\title{
NDE Evidence for the Damage Arrestment Performance of PRSEUS Composite Cube During High-Pressure Load Test
}

\author{
Patrick H. Johnston and F. Raymond Parker \\ NASA Langley Research Center \\ Hampton, Virginia 23681
}

\begin{abstract}
As an approach to light-weight, cost-effective and manufacturable structures required to enable the hybrid wing body aircraft, The Boeing Company, Inc. and NASA have developed the Pultruded Rod Stitched Efficient Unitized Structure (PRSEUS) concept. A PRSEUS pressure cube was developed as a risk reduction test article to examine a new integral cap joint concept as part of a building block approach for technology development of the PRSEUS concept. The overall specimen strength exceeded the 18.4 psi load requirement as testing resulted in the cube reaching a final pressure load of around 48 psi prior to catastrophic failure. The cube pressure test verified that the joints and structure were capable of sustaining the required loads, and represented the first testing of joined PRSEUS structure. This paper will address the damage arrestment performance of the stitched PRSEUS structure. Following catastrophic failure of the cube, ultrasonic pulse-echo inspection found that the localized damage, surrounding a barely-visible impact damage site, did not change noticeably between just after impact and catastrophic failure of the cube, and did not play a role in the catastrophic failure event. Ultrasonic inspection of the remaining intact cube panels presented three basic types of indications: delaminations between laminae parallel to the face sheets, lying between face sheet and tear strap layers, or between tear strap and flange layers; delaminations above the noodles of stringers, frames or integral caps, lying within face sheet or tear strap layers; and delaminations between the laminae in the inner fillets of the integral caps, where pulloff stresses were expected to be highest. Delaminations of all three types were predominantly contained by the first row of stitches encountered. For the small fraction of delaminations extending beyond the first row of stitches, all were contained by the second stitch row.
\end{abstract}

\section{INTRODUCTION}

Under the Environmentally Responsible Aviation Project (ERA), the National Aeronautics and Space Administration (NASA) is working with The Boeing Company, Inc. toward developing new structural concepts for future aircraft to meet more environmentally friendly requirements. One of these new concepts is the Pultruded Rod Stitched Efficient Unitized Structure (PRSEUS) [1]. The PRSEUS concept, depicted in Fig. 1, is being developed as a low-cost, light-weight composite structure for aircraft, which offers advantages over traditional metallic structure. The PRSEUS concept comprises a stitched carbon-epoxy material system with the potential for reducing the weight and cost of transport aircraft structure by eliminating fasteners, thereby reducing part count and labor. By adding unidirectional carbon rods to the top of stiffeners, the panel becomes more structurally efficient. A key feature of PRSEUS is the crack-arresting nature of the stitches, which enables the use of fail-safe design principles.

In recent years, several mechanical tests of the PRSEUS structure have been conducted [2-8], where ultrasonic pulse-echo testing (UT) was employed to monitor sites of intentional barely visible impact damage (BVID) for growth caused by loading. Additionally, in one test, a substantial section of the test article remained intact after catastrophic failure, permitting a post-mortem UT survey of large areas of the article. This paper presents UT results from those studies, which provide evidence of the damage-arresting performance of the stitching in those PRSEUS test articles.

\section{PHASED ARRAY ULTRASONIC MEASUREMENTS}

Interrogation of the PRSEUS panels was performed using normal-incidence phased array ultrasonic techniques (PAUT). The arrays were commercial 64-element (each 0.4" x 0.02"), $10 \mathrm{MHz}$ linear arrays, either mounted on a solid plastic $0^{\circ}$ wedge (for flat surfaces) or in a water-filled housing with rubber membrane (for curved surfaces or surfaces with strain gages and their associated wiring installed for test) [3]. A spray of water on the panel surface served as ultrasonic couplant. Effective apertures comprising groups of 16 adjacent elements were electronically scanned by 1-element spacing along the length of the array, and the probe was mounted to a manual X-Y manual 
scanner for positioning, as shown in Fig. 2. Pulse-echo signals were measured at each position over the scanned area of up to $20 " x 20 "$.
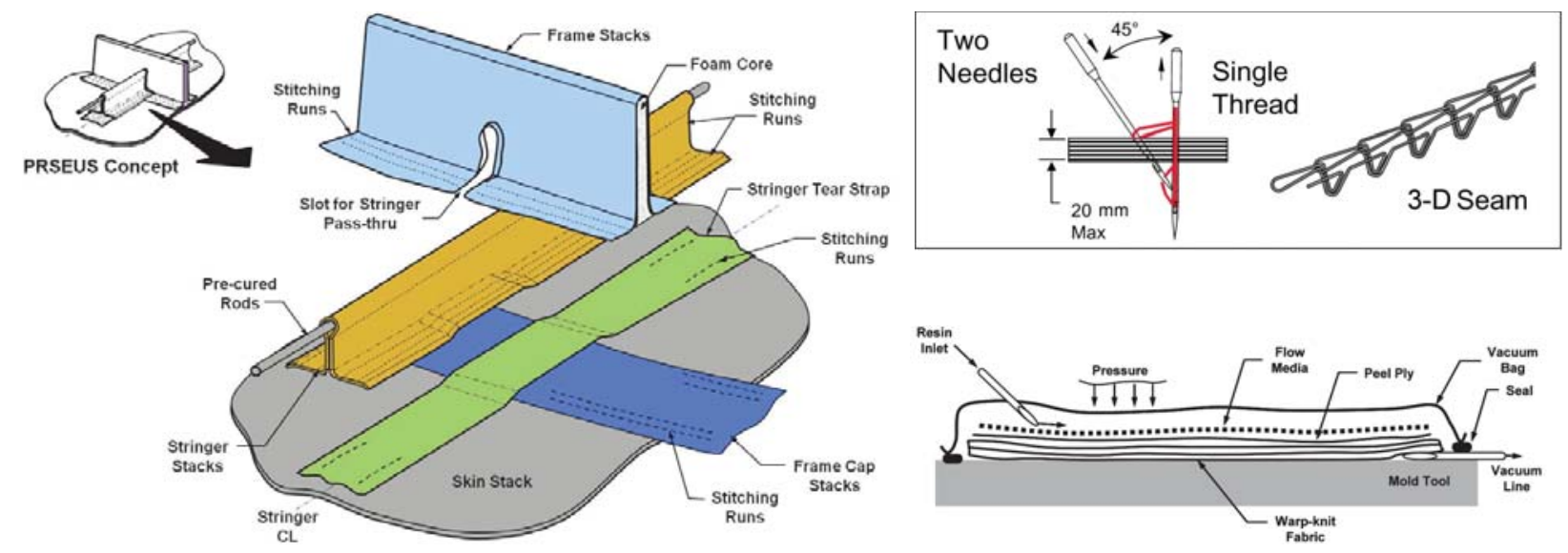

FIGURE 1. The PRSEUS dry preform is built up from carbon fabric stacks for the frame, stringers and skin. The layers are stitched together with Vectran fibers, using a single-sided sewing method, then bagged and infused with resin [1].

\section{TEST ARTICLES}

This paper will present results from three different test articles: a flat, 7-stringer and 4-frame panel, loaded in compression first statically, then cyclically [2,3]; a curved, 7-stringer and 5-frame panel, loaded in axial tension and pressure with reactive edge loads [4]; and a cube, comprising six flat PRSEUS panels joined via integral caps along their edges, loaded in pressure, ultimately to catastrophic failure [5-8].

\section{Linear array with solid plastic delay}

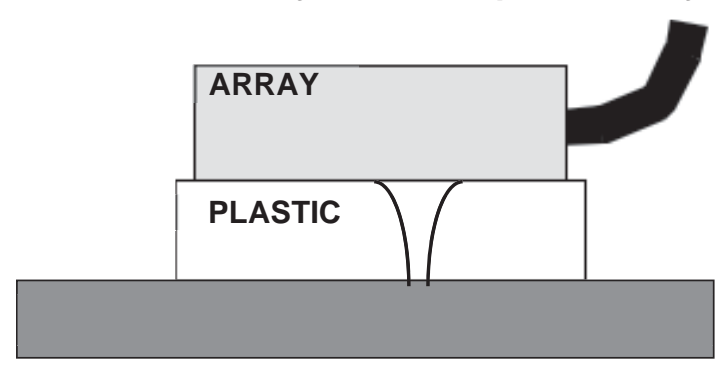

Linear array with water delay
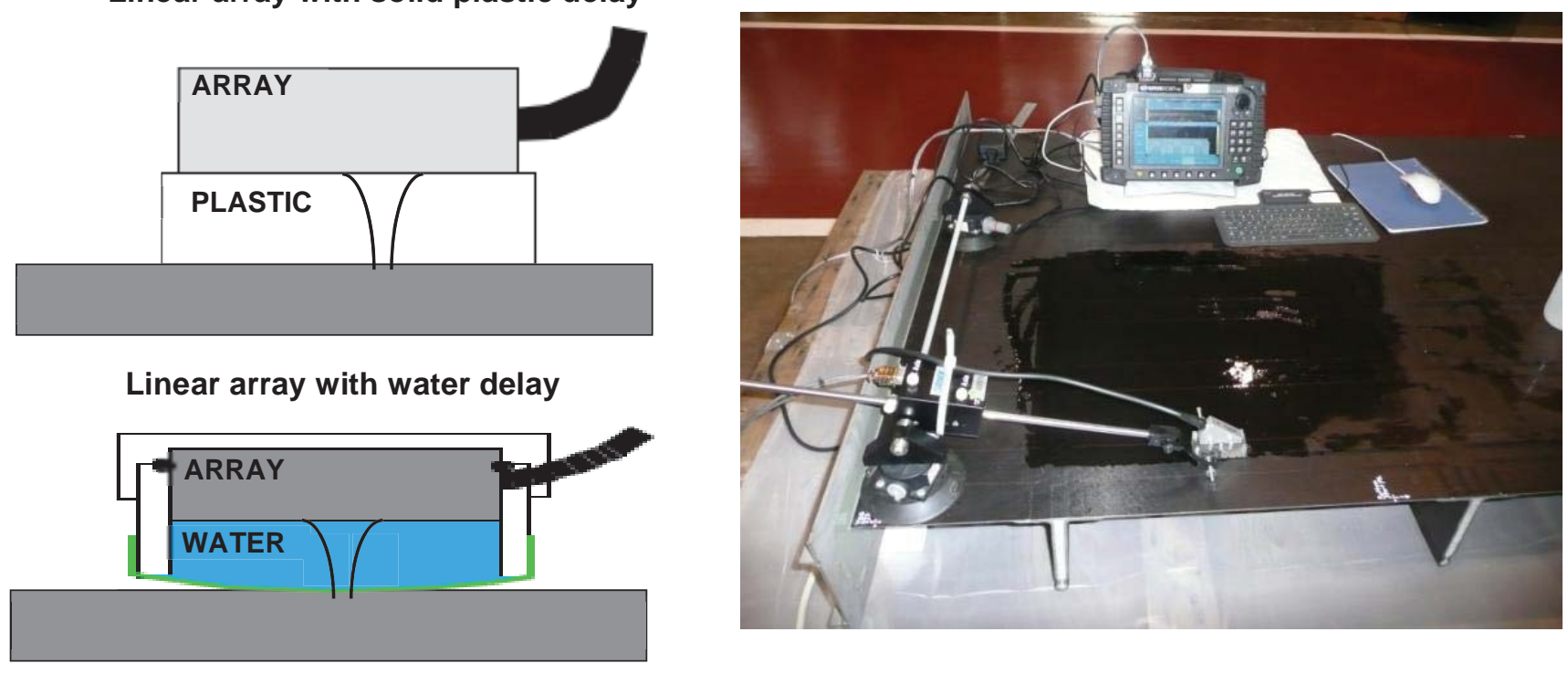

FIGURE 2. A linear ultrasonic array coupled to a manual X-Y encoder was used to interrogate PRSEUS panels. On flat panels, without gages, a solid plastic wedge was used. In order to better accommodate the presence of wires, gages, and tape, a captive water column with flexible face was used after panel instrumentation. The captive water probe was also used on the curved panel. 


\section{RESULTS}

\section{Flat 7-Stringer Panel}

The results for this panel were previously reported at the 2010 QNDE conference [3]. They are included here for comparison with the results obtained from the two later tests. Figure 3 presents the PAUT pulse-echo C-scan results at various stages of the test. In all panels, the grayscale represents the signal amplitude at the depth corresponding to the back surface of the free skin, so the back surface of the skin is gray, while the thicker flange regions are darker. Panels 3(a) and 3(b) show scans of the impact area before and after impact, respectively. A delamination is observed between the skin plies and the underlying flange plies, which runs vertically approximately 4" above and 4" below the impact point, while being held constrained in the horizontal direction by the first line of stitches encountered.

Figure 3(c) shows the C-scan obtained after the panel was loaded in static compression to its design limit load of 137,000 pounds. The dimensions of the delamination remained unchanged. Figure 3(d) presents the C-scan obtained after the panel was subjected to 20 cycles of compression up to 96,000 pounds. Again, the dimensions of the delamination at the BVID point remained unchanged.

\section{Curved 7-Stringer Pressure Panel}

The second test was performed in collaboration with the Federal Aviation Administration (FAA), utilizing the Fullscale Aircraft Structure Test Evaluation and Research (FASTER) Facility located at the FAA Technical Center in Atlantic City, NJ [4]. This test frame emulates the loads encountered by pressurized aircraft fuselage sections. In this test, a curved PRSEUS panel was subjected to hydrostatic pressure, along with reactive tensile loading at the edges,

(a) C-scan Pre-Impact

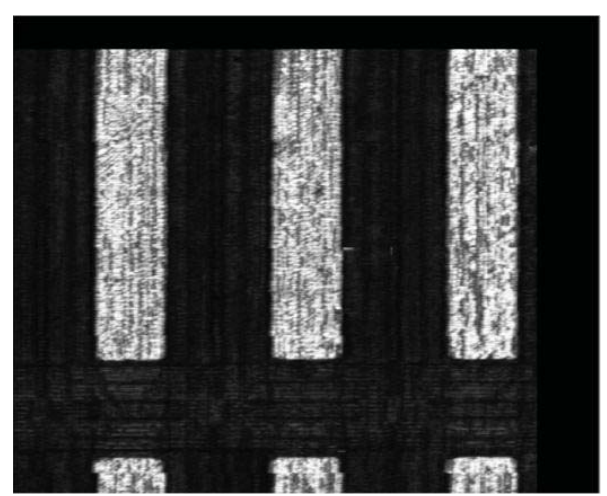

(c) C-scan Post-Static to Limit Load

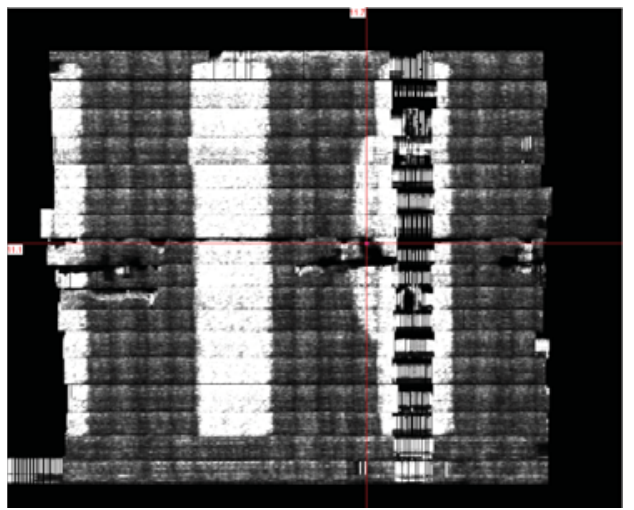

(b) C-scan Post-Impact

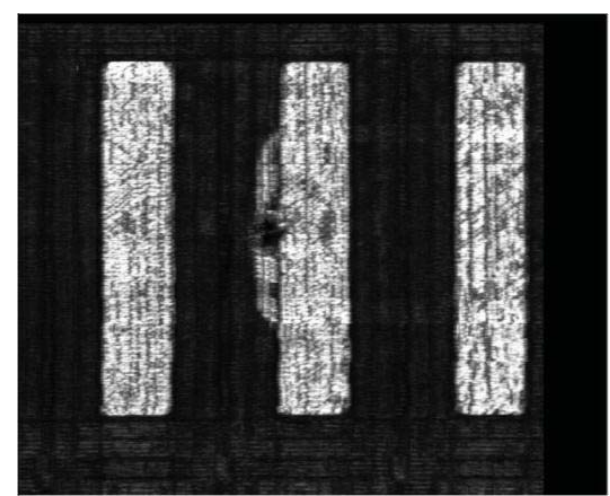

(d) C-scan Post-Cyclic Load

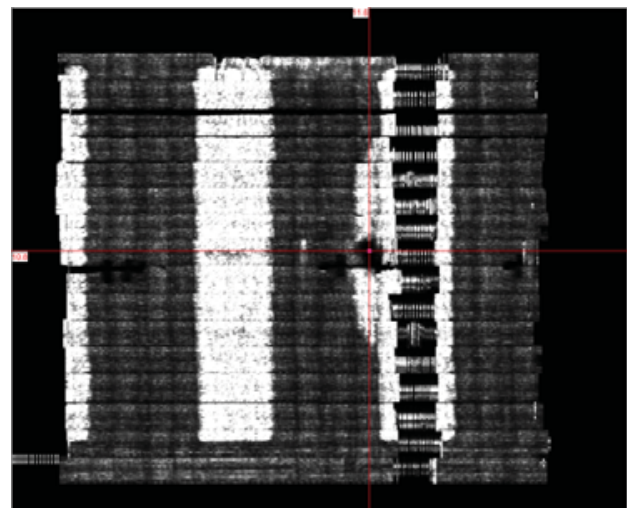

FIGURE 3. C-scans of the flat 7-stringer panel before (a) and after BVID (b), after static loading to limit load (c), and after 20 cycles of compression loading (d). 
and axial tensile loads. Phased array ultrasonic testing was periodically employed to monitor a site of BVID near the center of the panel.

Ultrasonic C-scans of the BVID area before and after impact are presented in Fig. 4. There is a considerable amount of artifacts in these scans, caused by difficulty in performing a manual scan within the constraints of the load frame, but these could be distinguished from actual echoes by examining the A-scan waveforms. One such artifact is present in the center of Fig. 4(b). A-scan analysis showed that this area was free from delamination. The scan post-impact is presented in Fig. 4(c), and also in Fig. 4(d) with the addition of a cartoon outline to highlight the delamination area. The formed delamination is similar to that observed in the flat compression panel, Fig. 3(c), with an additional small area of delamination between the first two stitch rows.

Subsequent to applying BVID, the panel was subjected to axial tension up to design limit load, pressure to design limit load, combined axial tension and pressure to design limit loads, axial tension to design ultimate load, pressure to design ultimate load, and combined axial tension and pressure to design ultimate loads. After each of these test conditions was achieved, a C-scan was performed over the area containing BVID. These C-scans are presented in Fig. 5. Despite the presence of some variation in coupling, it was observed that the original delamination area did not grow, either in length or across the stitches.

After these tests, a saw cut was made through the stringer, passing through the BVID point, and the panel was pressure loaded to failure. The damage to the panel was widespread, and no further C-scans were performed.

(a) Curved Panel

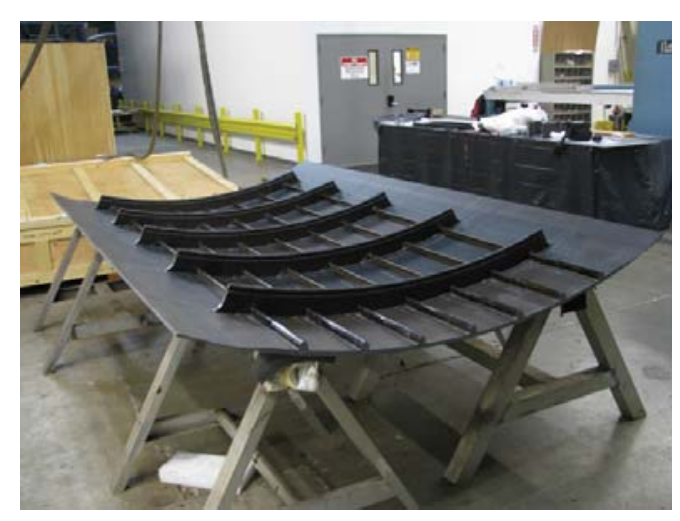

(c) C-scan Post-Impact

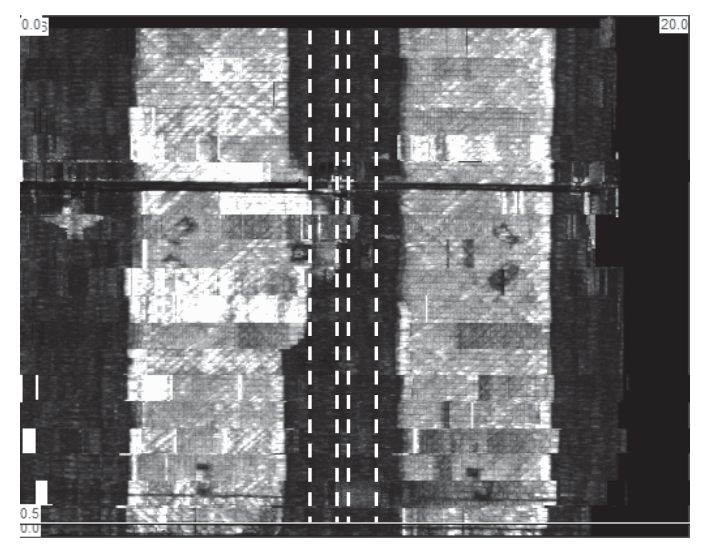

(b) C-scan Pre-Impact

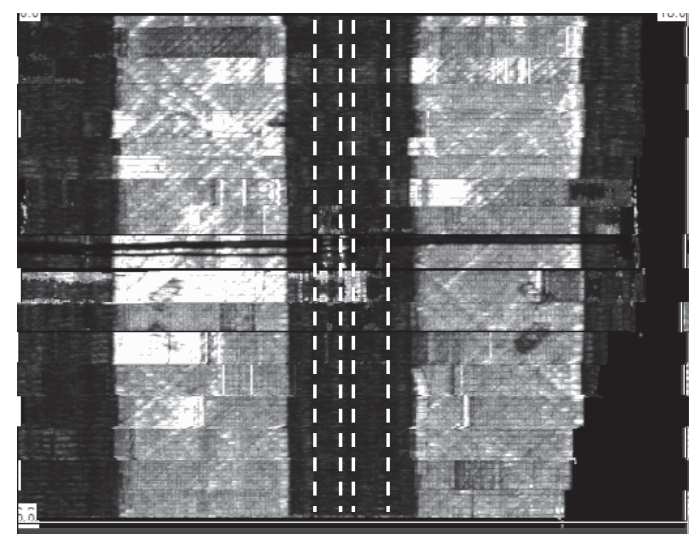

(d) C-scan Post-Impact with Highlight

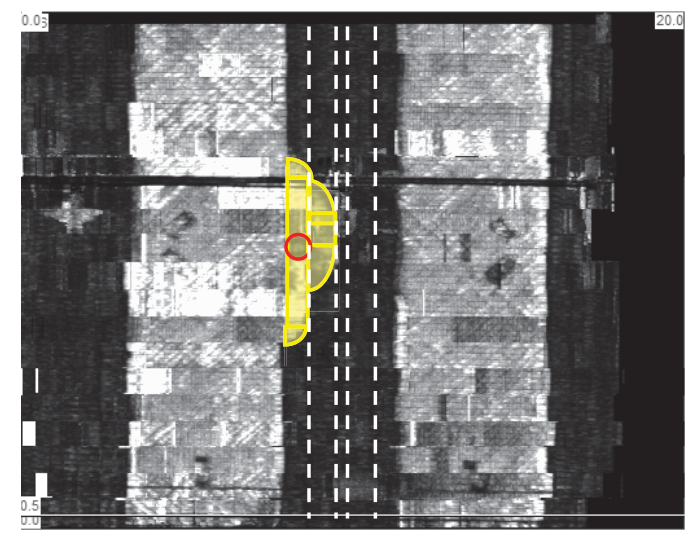

FIGURE 4. (a) Photograph of the curved test panel. (b) C-scan of panel before BVID. (c) C-scan of panel after BVID. (d) Post-impact C-scan with a cartoon outline to highlight the delamination and the impact point (circle). 

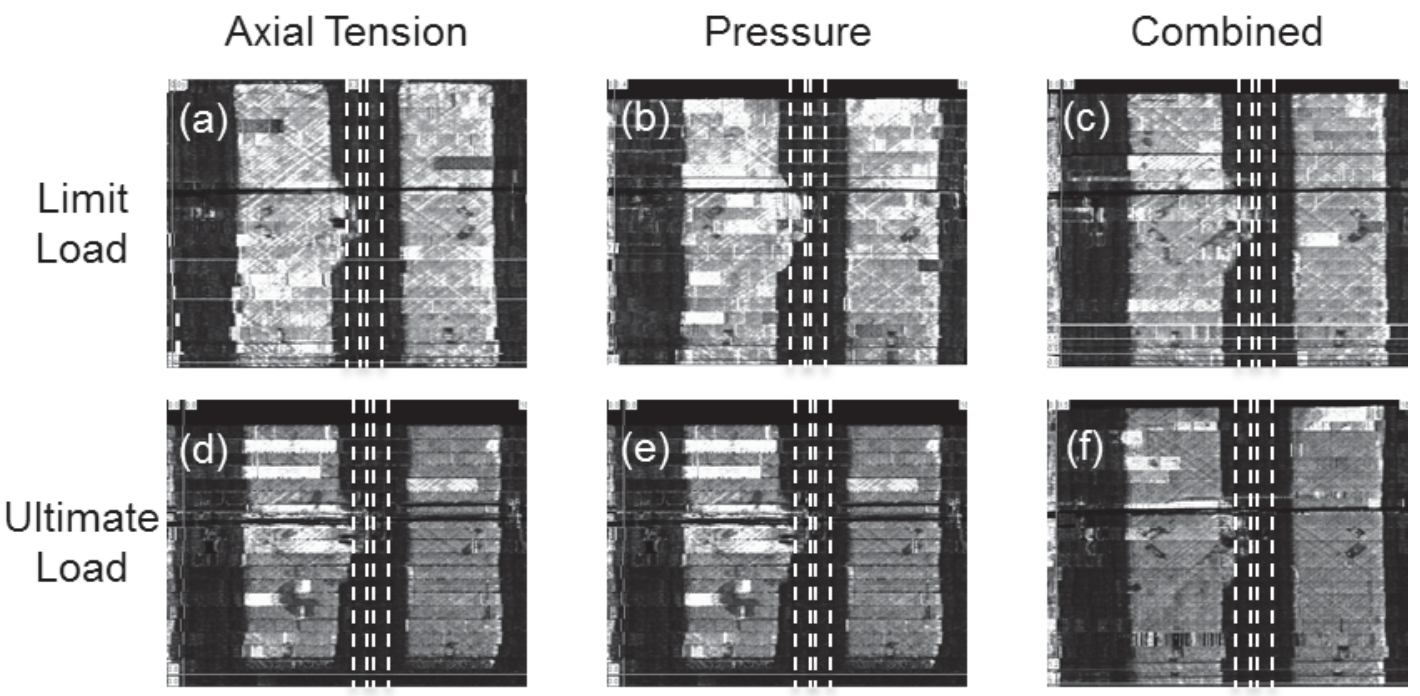

FIGURE 5. C-scans of the BVID area on the curved panel were performed following six different loading conditions. The delamination remained unchanged after all loading conditions. The stitch rows are indicated by dashed lines.

\section{Pressure Cube}

The final test article was a pressure cube, comprising six flat PRSEUS panels, joined to form a sealed, nearly cubical structure [5-8]. A graphic of the cube is shown in the inset of Fig. 6, with one side removed to show the interior details. The cube test served as a demonstration of the joining concept, planned for a much larger mostlyPRSEUS multibay test article currently being fabricated by Boeing. As with the earlier tests, the cube was subjected to BVID, and PAUT inspection identified a minimal amount of damage around the impact site, which remained unchanged throughout the pressure tests. Various stages of pressure were applied, with the final stage being pressurization to failure. Success of the joint test was assured at 18.4 psi pressure, twice the normal operating pressure for an aircraft, but the structure remained intact until $48 \mathrm{psi}$, when a metallic component of a corner joint began to crack, after which the forward bulkhead panel failed catastrophically. The crown, floor, and other three sides of the cube remained intact, allowing subsequent ultrasonic scans to be performed.

Scans of the remaining panels detected three distinct types of delaminations, distinguished by their locations in the structure. The drawing in Fig. 6 illustrates these three types, labeled A, B, and C. Type A delaminations occur between the laminae of the face sheet, tear or cap strap, and flange layers. The delaminations found in the flat 7stringer panel and curved panel were of type A. The second delamination type B was observed in the skin or strap layer directly above a perpendicular web component, be it a stiffener, a frame, or an end cap. The final damage type $\mathrm{C}$ appeared in the inboard flange layers of end caps adjacent to the interior fillet. Figure 6 includes a B-scan data set of an integral cap to show an example of Type C damage and to illustrate the locations of Type A and Type B delaminations.

A summary of the results for the crown panel is given in Fig. 7. The results for the aft bulkhead and the right and left rib panels are similar in character. The metallic fitting which is believed to have initiated failure was located in the joint at the lower left corner of this figure. This will be referred to as corner I.

In the crown, three areas of Type A delamination were observed, near corner I and along the joint with the failed forward bulkhead. Similarly, on the right rib panel significant areas of Type A damage were along the joint with the forward bulkhead and near corner I. These delaminations were observed to cross stitch rows in places. Because of their location, the Type A delaminations are believed to have formed due to rapid, high intensity load redistribution during the time period between failure initiation in corner I and the final catastrophic failure of the forward bulkhead.

Type B delaminations are observed in Fig. 7 to occur in all of the stringers, extending the entire length between frames, and in the frame ends near each corner. The Type B damage is contained between the central two stitch rows in all of these areas.

In the crown panel, Type $\mathrm{C}$ delaminations were observed along most of the length of the inner flange of the integral cap. Inboard, the observed Type $\mathrm{C}$ flaws did not extend beyond the second stitch row. However, because of 
the ultrasonic "shadow" above the cap webs (Fig. 6), the extent of these delaminations into the curved fillet was unknown.

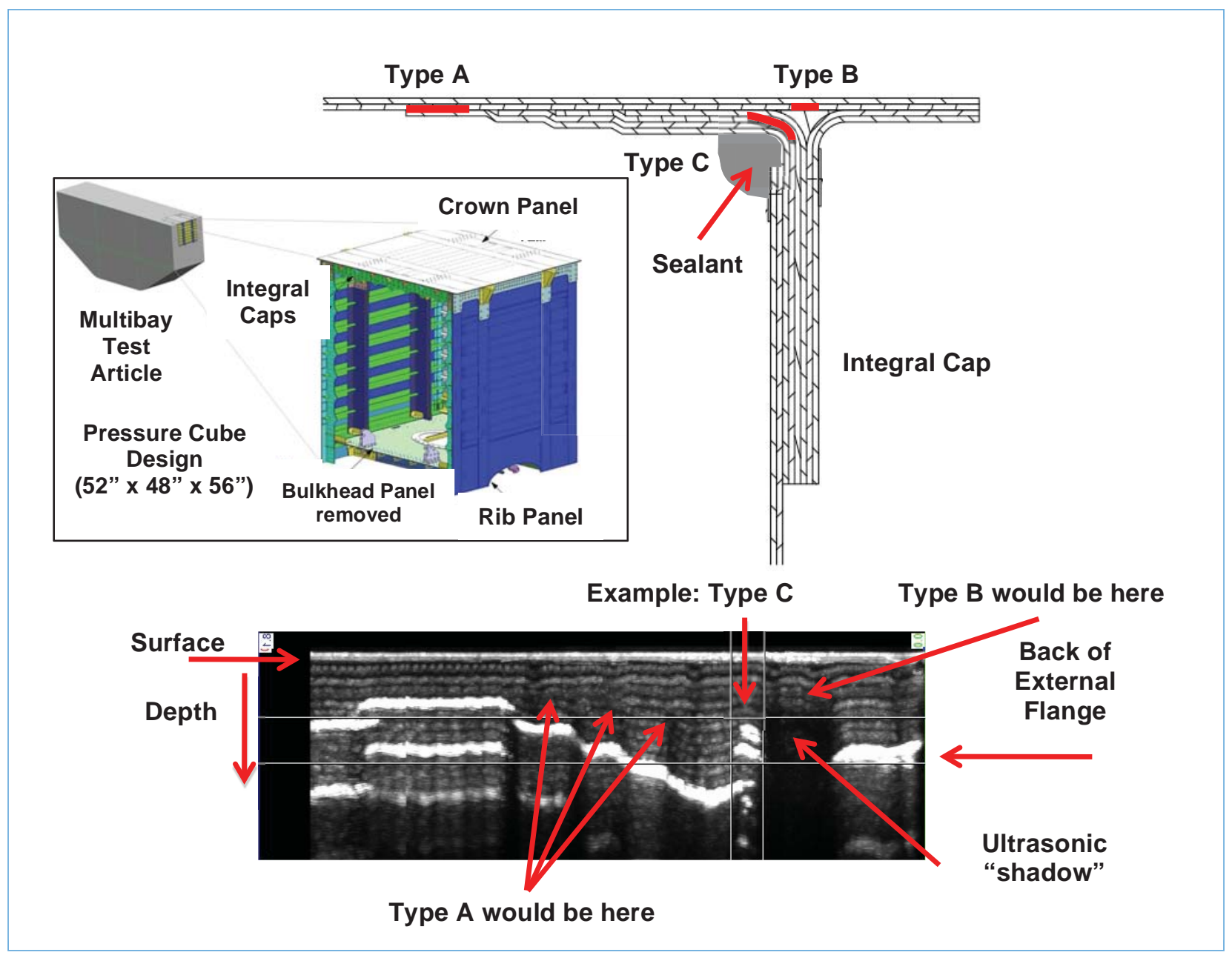

FIGURE 6. The PRSEUS pressure cube (inset) was designed to demonstrate the joint design intended for use on the full large-scale multibay test article. After loading the cube to failure, three types of delaminations were identified, based on location. Type A occurred between face sheet and flange plies; Type B delaminations occurred in the plies above the noodle of reinforcing structures. Type $\mathrm{C}$ delaminations occurred in the flange plies adjacent to the inner fillet of the integral cap webs. The fillet region was masked by an ultrasonic "shadow, believed to be caused by the noodle.

In order to address that question, a section of the crown containing an integral cap was excised for immersion UT and x-ray computed tomography (CT). Immersion C-scans of the skin side and the now-accessible web of the excised cap are shown in Fig. 8. From the skin side, the results are the same as obtained earlier using the phased array. The observable Type $\mathrm{C}$ delaminations are contained between stitch rows. Similarly, in the web, some Type C delaminations are observed past the first stitch row, but do not pass the second row. A CT image of the fillet region (Fig. 9) shows that the curved inner fillet is highly delaminated, resembling the layers of an onion. This condition is consistent with the presence of high out-of-plane stresses in the fillet, predicted by analysis, during pressurization. Almost all of these delaminations are seen to terminate at or inside the first stitch encountered, either the diagonal stitch in the face sheet or the normal stitch in the web. The few delaminations which do pass the first stitch row are observed to end before the next stitch is encountered. 


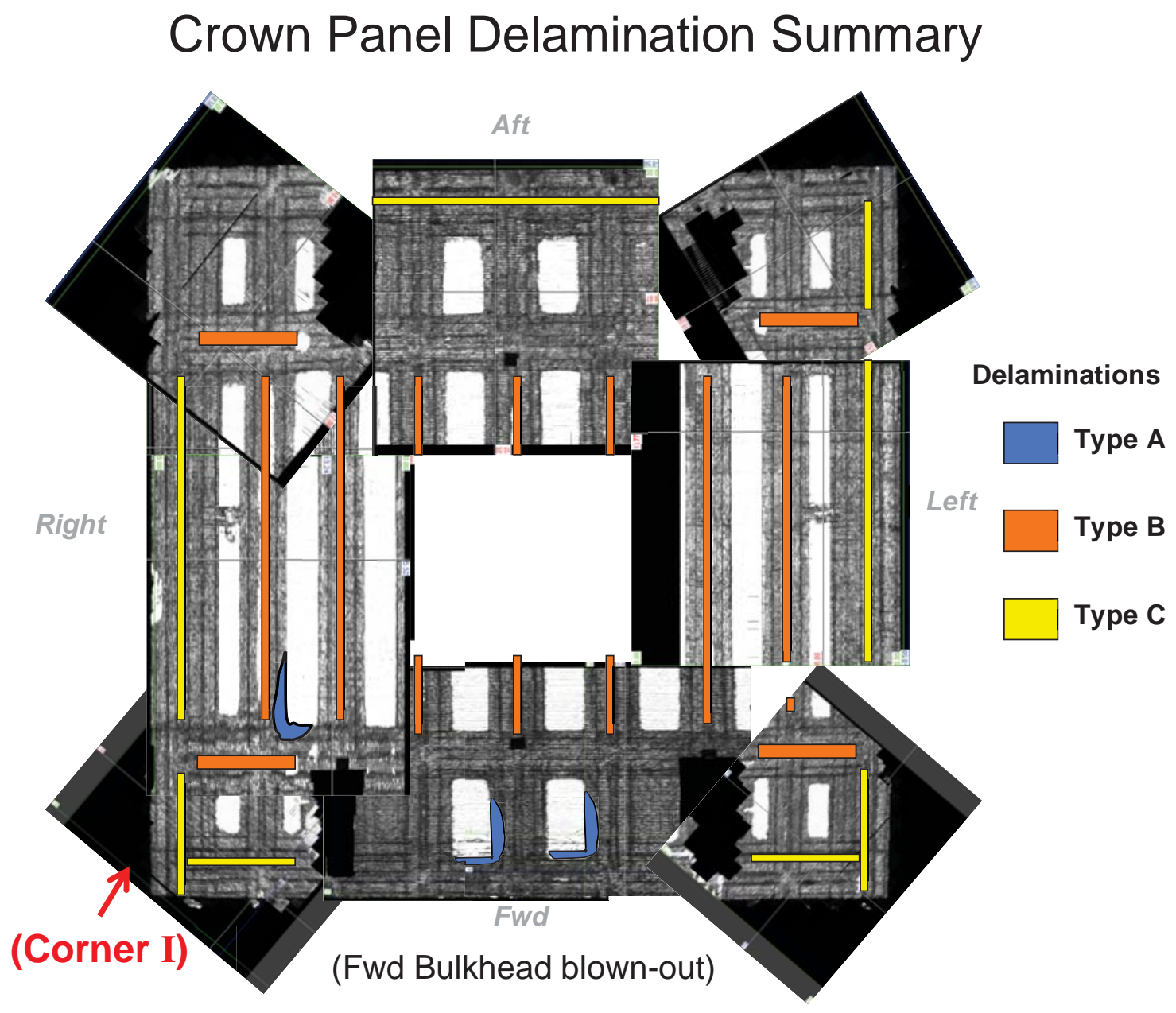

FIGURE 7. A summary diagram of the delaminations observed in the crown panel.

\section{CONCLUSIONS}

Ultrasonic NDE of PRSEUS test articles during load tests have demonstrated that delamination damage, which is formed in response to BVID or which occurs due to pressure loading, is contained by the rows of stitches in the material. This behavior supports the argument that PRSEUS enables the use of fail-safe design principles.

\section{REFERENCES}

1. A. Velicki, NASA-CR-2009-215932 (2009).

2. D. C. Jegley, Proceed. Soc. of Exper. Mech. Conf., Mohegan Sun, Uncasville, CT (2011).

3. P. H. Johnston, Rev. Prog. Quant. Nondestr. Eval., 30B, 1432-1439 (2011).

4. A. Bergan, et al, $2^{\text {nd }}$ Annual Aircraft Airworthiness \& Sustainment Conference (2011).

5. N. Yovanof, et al, $3^{\text {rd }}$ Annual Aircraft Airworthiness and Sustainment Conference, (2012).

6. A. E. Lovejoy, NASA-TM-2013-217795 (2013).

7. M. R. Horne and E. I Madaras, NASA-TM-2013-217993 (2013).

8. P. H. Johnston, NASA-TM-2013-217799 (2013). 


\section{Immersion C-Scans of Cutout End Cap}

(a)

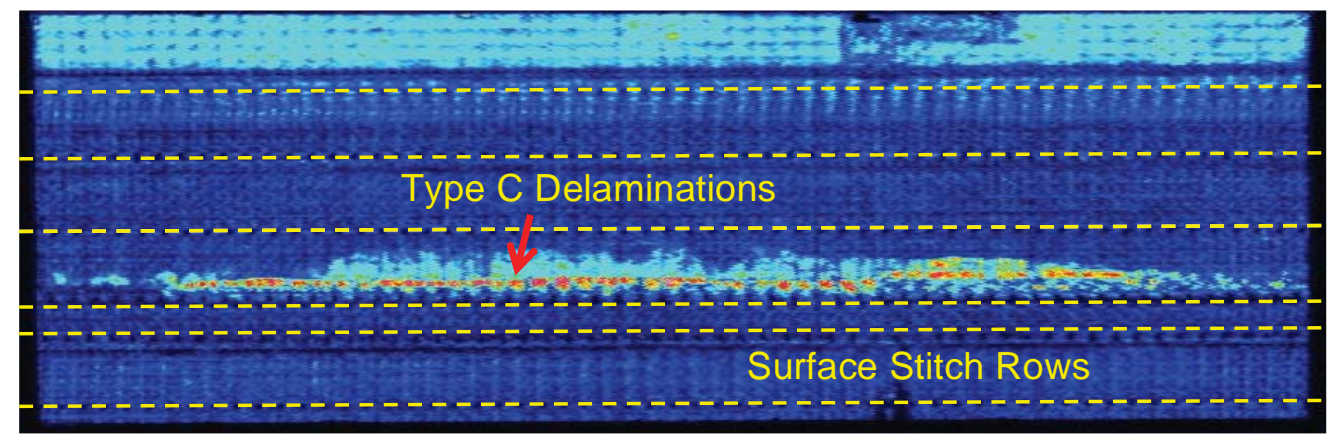

(b)

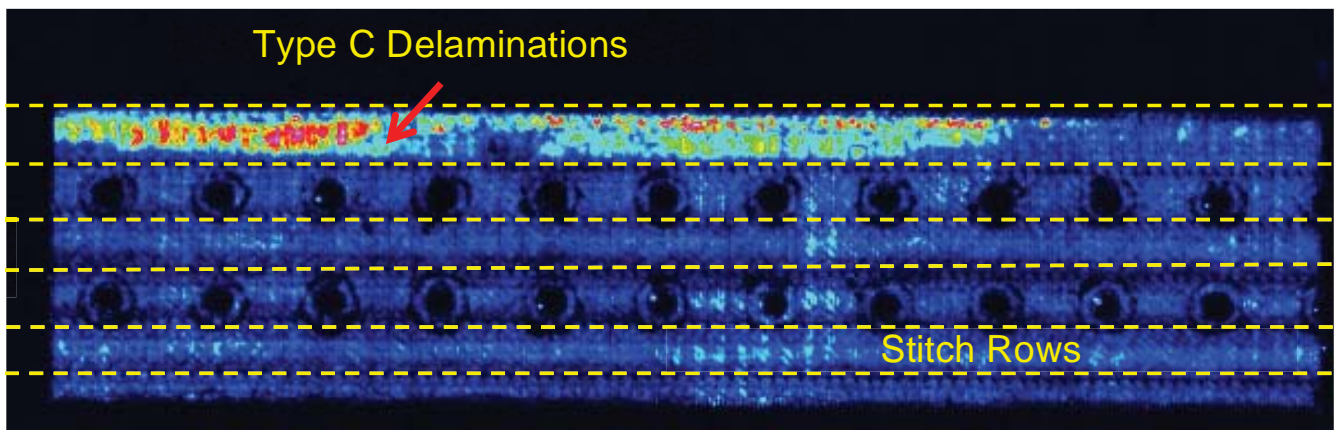

FIGURE 8. Immersion C-scans of the skin (a) and the web (b) of a section cut from the crown including an integral cap.

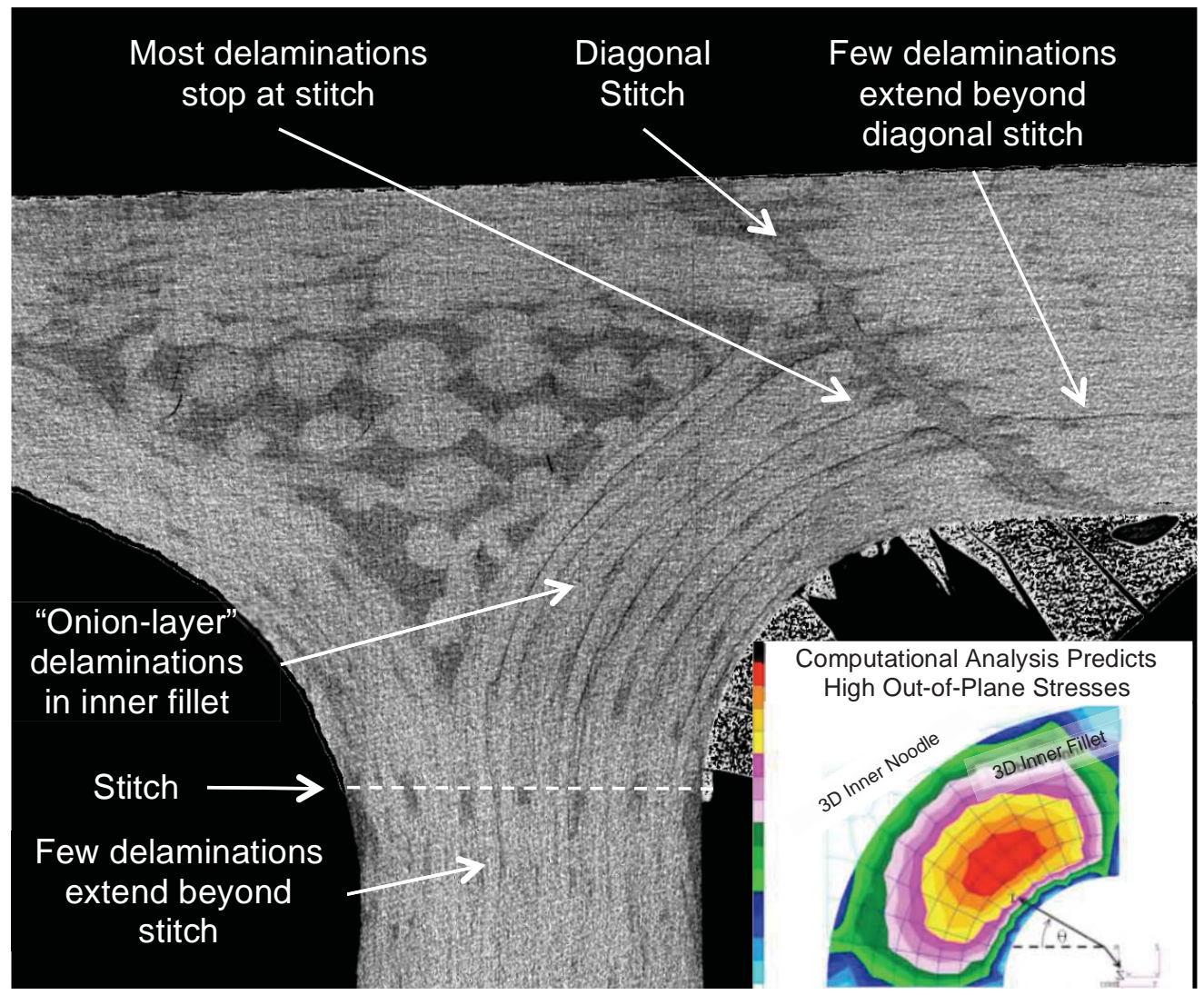

FIGURE 9. (a) A CT slice of the noodle area of the crown integral cap, showing the highly delaminated fillet plies, relative to the stitches. (b) Computational analysis predicted high out-of-plane stresses in the fillet during pressurization, consistent with the presence of the "onion-layer" delaminations in the fillet. 